\title{
Analysis on the influence of $\mathrm{ZnO}$ addition on microwave dielectric properties of $\mathrm{Li}_{2} \mathrm{MgSiO}_{4}$ ceramics
}

\author{
ALEENA ROSE ${ }^{1}$, B MASIN ${ }^{2}, K_{\text {ASHOK }}{ }^{2}$, H SREEMOOLANADHAN ${ }^{2}$ and T VIJAYAKUMAR ${ }^{1, *}$ \\ ${ }^{1}$ Futuristic Materials Research Centre for Planetary Exploration, Department of Physics and Nanotechnology, Faculty of \\ Engineering and Technology, SRM Institute of Science and Technology, Kattankulathur, Kancheepuram 603203, India \\ ${ }^{2}$ Advanced Materials and Ceramic Division, Vikram Sarabhai Space Center, Thiruvananthapuram 695022, India \\ *Author for correspondence (vijayakumar.t@vdp.srmuniv.ac.in)
}

MS received 12 October 2018; accepted 14 February 2019

\begin{abstract}
Dielectric ceramics have gained wide attention owing to their applicability in a broad range of domains including a microwave communication system. Silicates are considered as the prospective substrate materials for microwave circuits due to their properties complementing with the requisites of a microwave substrate. Lightweight lithium-based silicate ceramic namely lithium magnesium silicate $\left(\mathrm{Li}_{2} \mathrm{MgSiO}_{4}, \mathrm{LMS}\right)$ was prepared by a solid-state reaction method. Zinc oxide $(\mathrm{ZnO})$ was used as an additive to LMS in different weight percentages $(0.5,1.0,1.5$ and 2.0) and LMS-ZnO ceramics were also prepared. The microwave dielectric properties of pure LMS and ZnO-added LMS were measured and the influence of $\mathrm{ZnO}$ on LMS was investigated. A dielectric resonance technique was utilized to study the dielectric properties of ceramic samples at $12 \mathrm{GHz}$. A comparatively higher quality factor of $24,000 \mathrm{GHz}$ was attained by the LMS-ZnO (2 wt $\%)$ system with a relative density of $90.2 \%$ and $<10$ dielectric constant of 5.76 making it suitable for substrate applications.
\end{abstract}

Keywords. Solid-state reaction; silicates; dielectric ceramics; microwave; quality factor.

\section{Introduction}

Microwaves of the electromagnetic spectrum, residing between radio and infrared waves have been widely explored in the communication field owing to their advantages over radio waves. They can be easily focussed onto narrower beams which allow frequency reuse and relatively higher frequency allocates a wide bandwidth and provides a high-data transmission rate. Since the antenna size is inversely proportional to the transmitted frequency, the antenna size can be reduced. Requisite of a good communication system i.e., transmission of energy and information signals with no interference can be achieved through the use of microwaves. They travel by line of sight propagation and do not follow the Earth's curvature. Large reflections are produced because of their short wavelength. The wavelength similar to the circuit dimensions initiates the use of waveguides and cavity resonators replacing the coaxial transmission lines and lumped-element tuned circuits [1-3]. All these factors increased the use of microwaves in point-to-point telecommunications, radar, remote sensing, satellite communication, tracking applications, etc. Thus, the development of microwave communication field strengthened the exigency for microwave ceramics which possess good dielectric properties, and ability to be incorporated with other materials $[4,5]$. These highly reliable ceramic systems are utilized for a wide range of applications including antennas, packages, substrates, cell phones, capacitors, filters, resonators, etc. [6-8]. Relative permittivity $\left(\varepsilon_{\mathrm{r}}\right)$ or dielectric constant, dielectric loss $(\tan \delta)$ or quality factor (Quxf) and temperature coefficient of resonant frequency $\left(\tau_{\mathrm{f}}\right)$ are the three important parameters which should be optimized for a specific application [9]. Low-loss ceramics with low-dielectric constant values $(<10)$ and near-zero temperature coefficient of the resonant frequency are of great significance owing to their potential to be used as substrate materials in microwave integrated circuits. A substrate material with low $\varepsilon_{\mathrm{r}}$ and $\tan \delta$ aids the signal transmission by decreasing the noise, propagation delay and signal attenuation, respectively [10-13]. Additionally high Quxf provides selectivity through discarding the unnecessary signals by its narrow bandwidth [14]. Microwave dielectric ceramics such as silicates, aluminates and phosphates are capable of meeting these requirements [15-17]. Lithium magnesium silicate $\left(\mathrm{Li}_{2} \mathrm{MgSiO}_{4}\right)$, forsterite $\left(\mathrm{Mg}_{2} \mathrm{SiO}_{4}\right)$, wollastonite $\left(\mathrm{CaSiO}_{3}\right)$, zinc silicate $\left(\mathrm{Zn}_{2} \mathrm{SiO}_{4}\right)$, etc. are some of the silicates exhibiting low $\varepsilon_{\mathrm{r}}$ and $\tan \delta$ which makes them suitable candidates for microwave substrate applications [5,18-20].

Density has a huge impact on the dielectric properties of ceramics. The lack of densification limits the application of silicate compounds in microwave-integrated circuits. The development of a densified ceramic depends on the crystal structure, defects, pore density, fabrication route and the characteristics and concentration of additives and dopants [21]. Porosity, micro-cracks, non-uniform grain growth, grain boundaries, crystal imperfections, etc. reduce the density of the ceramics thereby leading to extrinsic loss [14]. 
Inclusion of additives or dopants is the widely accepted method to improve the density of a ceramic material. $\mathrm{CuO}$, $\mathrm{ZrO}_{2}, \mathrm{TiO}_{2}, \mathrm{MnO}_{2}, \mathrm{ZnO}, \mathrm{CaF}_{2}, \mathrm{PbF}_{2}, \mathrm{~B}_{2} \mathrm{O}_{3}$, etc. are some of the additives used for achieving good densification [22-26]. Among these additives, $\mathrm{ZnO}$ is reported to have an immense potential to enhance the microstructural density of dielectric ceramics. Tan et al [27] studied the effect of $\mathrm{ZnO}$ addition on the densification of forsterite ceramic and concluded that $\mathrm{ZnO}$ improved the density and microstructure of forsterite without inducing any chemical changes. Nikzad et al [28] achieved a less porous structure and high Quxf of $141,000 \mathrm{GHz}$ for $\mathrm{ZnO}$ added $\mathrm{MgTiO}_{3}$ at a relatively low-sintering temperature of $1050^{\circ} \mathrm{C}$. Research work by Lu et al [29] shows that an optimal concentration of $\mathrm{ZnO}$ in $0.90 \mathrm{Al}_{2} \mathrm{O}_{3}-0.10 \mathrm{TiO}_{2}$ can reduce the sintering temperature, lessen the number of pores and smoothen the grain size of that ceramics. Muccillo et al [30] obtained $94 \%$ of the theoretical density for yttrium-doped barium zirconate through $\mathrm{ZnO}$ addition. A recent study on $\mathrm{ZnO}-$ $\mathrm{H}_{3} \mathrm{BO}_{3}(\mathrm{ZH})$ addition to $\mathrm{ZnO}-\mathrm{MgO}-\mathrm{TiO}_{2}-\mathrm{CaO}$ (ZMTC) dielectric ceramics revealed the correlation between density, grain shape and dielectric properties of ZMTC and quantity of ZH. In this work, Guo and Zhang [31] achieved a Quxf of $60,000 \mathrm{GHz}$ for $8 \mathrm{wt} \%$ of ZH in ZMTC. Besides, $\mathrm{ZnO}$ has been used as a sintering aid in LTCC technology for decreasing the sintering temperature below the melting point of electrode materials through liquid phase sintering [32-34]. The aforementioned reports prove the ability of $\mathrm{ZnO}$ in promoting the densification and dielectric properties of microwave ceramics. Lithium is a lightweight metal. This type of material is highly appreciated for space applications because lightweight components can reduce the overall weight of satellite. Apart from this, lithium containing ceramic compositions show lowexpansion coefficients. But achieving a good quality factor with lithium-based ceramic compounds is difficult. As we have explained earlier, silicates are one of the compounds meeting the requisites for microwave substrate materials. Hence to obtain the synergistic effect, a silicate compound based on lithium is tried in this work.

Here, we have discussed the solid-state synthesis of $\mathrm{Li}_{2} \mathrm{MgSiO}_{4}$ (LMS) ceramics and impact of $\mathrm{ZnO}$ addition on the microwave dielectric properties of the LMS ceramic system. We have analysed the changes occurred in the density and dielectric properties of ceramics for various weight percentages of $\mathrm{ZnO}$.

\section{Experimental}

LMS and LMS-ZnO ceramic systems were synthesized through a four-step solid-state reaction method. Starting chemicals, $\mathrm{Li}_{2} \mathrm{CO}_{3}, \mathrm{MgO}$ and $\mathrm{SiO}_{2}$ (99.9\%, Aldrich Chemical Company), were preheated at $200^{\circ} \mathrm{C}$ for $1 \mathrm{~h}$ in order to remove the moisture content and impurities. Stoichiometrically weighed chemicals were mixed in a roll miller for $5 \mathrm{~h}$ by making use of agate balls and de-mineralized (DM) water.
This blend was then dried at $100^{\circ} \mathrm{C}$ for $2 \mathrm{~h}$. The resulting mix was ground to fine powder in an agate mortar and calcined at $850^{\circ} \mathrm{C}$ for $4 \mathrm{~h}$. A binder used for green body preparation was $1 \%$ polyvinyl alcohol $(\mathrm{MW} \sim 14,000)$ solution. Calcined LMS powder was mixed with this binder and cylindrical pellets of about $18 \mathrm{~mm}$ diameter and $5.9 \mathrm{~mm}$ thickness were made by applying a pressure of $100 \mathrm{MPa}$ in a manual press. Pure LMS was sintered at $1100^{\circ} \mathrm{C}$ for a duration of $6 \mathrm{~h}$.

LMS-ZnO systems were prepared for four different concentrations of $\mathrm{ZnO}$. Since $\mathrm{ZnO}$ was used as an additive, the particular weight percentage $(0.5,1,1.5$ and $2 \mathrm{wt} \%)$ of $\mathrm{ZnO}$ was added to calcined LMS powder and mixed thoroughly using DM water. Samples were then dried at $100^{\circ} \mathrm{C}$ for $2 \mathrm{~h}$ and ground to fine powder. Third step, green body preparation, was conducted similar to that of pure LMS. The diameter and thickness of LMS-ZnO pellets were 12 and $3 \mathrm{~mm}$, respectively. These ceramic systems were sintered at $1100^{\circ} \mathrm{C}$ for a duration of $4 \mathrm{~h}$.

Microstructural features of the sintered pellets were analysed by means of a FEI Quanta FEG 200-high resolution scanning electron microscope (HRSEM). Information about the crystal structure and phase purity of the samples was obtained from a CuK $\alpha$ Bruker D8 advance diffractometer. A BRUKER RFS 27: Stand-alone FT-Raman spectrometer was used to capture the Raman spectra of the samples. Density measurements and microwave dielectric properties of sintered ceramics were obtained through the Archimedes method and the dielectric resonance technique (TE01 $\delta$ mode) using a scalar network analyser (Aeroflex IFR6823) respectively.

\section{Results and discussion}

X-ray diffraction (XRD) patterns of LMS and LMS-ZnO ceramic systems are shown in figure 1. All the diffraction patterns shown in the figure are complaining with the ICDD

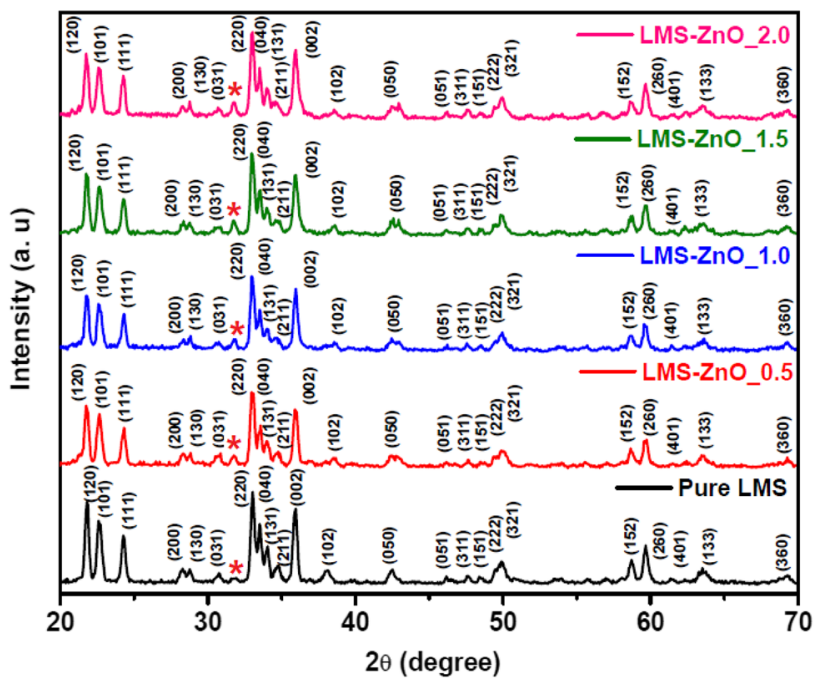

Figure 1. XRD patterns of pure LMS and LMS-ZnO ceramics. 

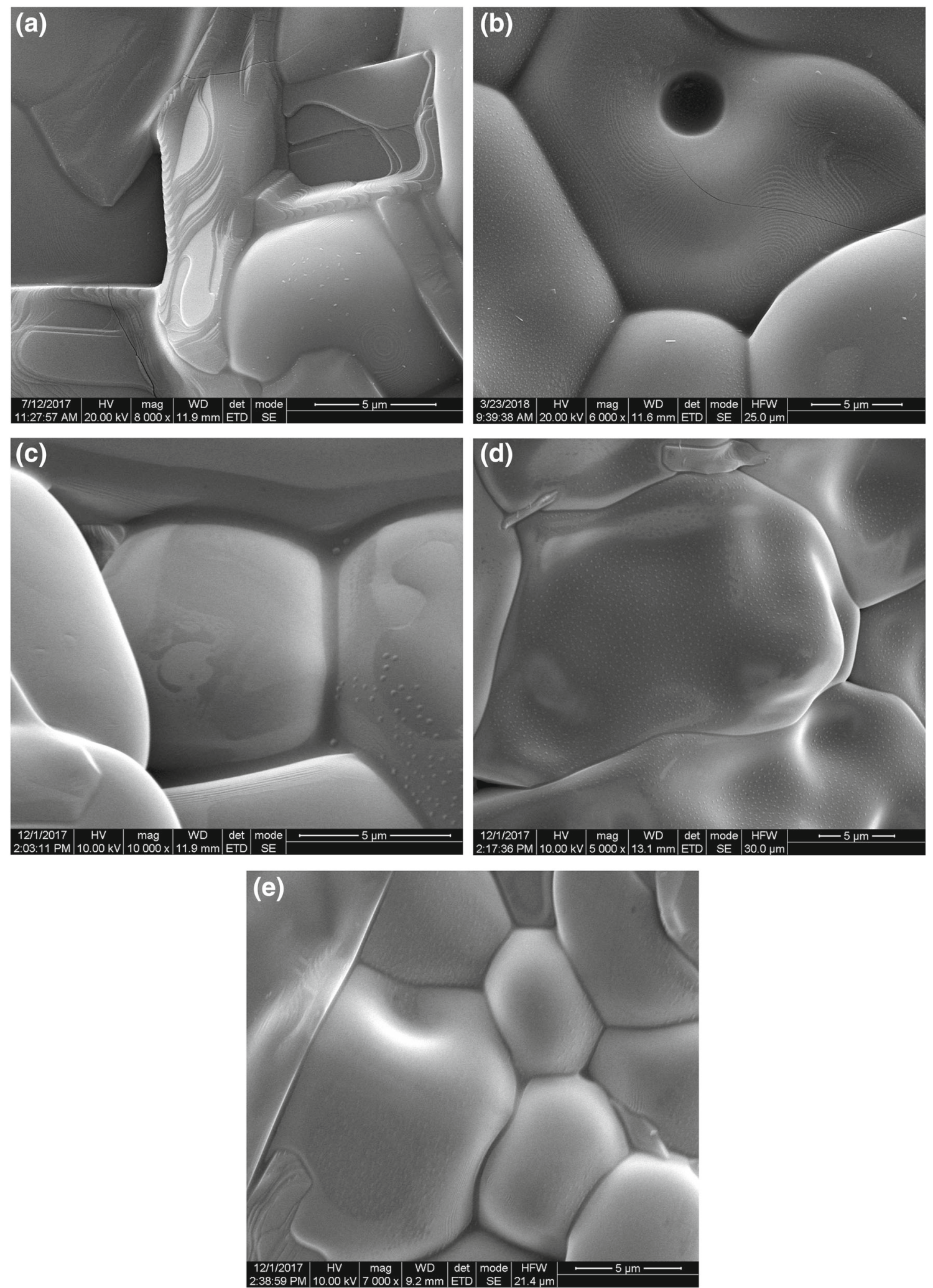

Figure 2. HRSEM of (a) pure LMS, (b) LMS-ZnO (0.5 wt \%), (c) LMS-ZnO (1 wt \%), (d) LMS-ZnO (1.5 wt $\%$ ) and (e) $\mathrm{LMS}-\mathrm{ZnO}(2 \mathrm{wt} \%)$ ceramic systems. 
pattern (024-0636) of LMS having an orthorhombic unit cell as the major phase. The minor secondary phase identified as lithium silicate $\mathrm{Li}_{6}\left(\mathrm{Si}_{2} \mathrm{O}_{7}\right)$ has been marked as '*' with very low-intensity reflection. The minor secondary phase, $\mathrm{Li}_{6}\left(\mathrm{Si}_{2} \mathrm{O}_{7}\right)-\mathrm{ICDD}$ (01-074-0300), is present in both pristine and $\mathrm{ZnO}$-added LMS patterns. It can be seen from figure 1 that addition of $\mathrm{ZnO}$ in LMS ceramic did not led to the formation of undesired phases or is not detectable in any of the XRD patterns.

Figure 2a-e shows the HRSEM of pure LMS and LMS$\mathrm{ZnO}$ ceramic systems with $0.5,1,1.5$ and $2 \mathrm{wt} \%$ of $\mathrm{ZnO}$ respectively. The microstructure of pure LMS contains more cracks and non-uniform grains in some areas. From figure $2 b$ it is clear that LMS-ZnO ( $0.5 \mathrm{wt} \%)$ shows cracks and trapped intra-granular pores along with large non-uniform grains. These features of microstructures may have a negative influence on the dielectric properties of LMS such as extrinsic loss. In the case of LMS-ZnO (1wt\%), figure $2 \mathrm{c}$, cohesive neck formation is complete but grains of non-uniform sizes and inter-granular pores are there. LMS-ZnO (1.5 wt \%) ceramic shown in figure $2 \mathrm{~d}$ consists of uniform large grains and completely grown cohesive necks compared to previously mentioned samples. The image of LMS-ZnO ( $2 \mathrm{wt} \%)$ captured by HRSEM as shown in figure 2e reveals its better refined microstructure and regular grain size. $\mathrm{ZnO}$ addition to LMS ceramics appears to be beneficial in refining the microstructure in terms of grain size and uniformity. Compared to other samples, energy dissipated by this ceramic may be low thus leading to a high-quality factor and low-loss tangent. None of the microstructures showed signs of grainboundary segregation which might be due to the solubility of $\mathrm{ZnO}$ in the LMS system.

Fourier transform (FT)-Raman spectra of LMS and ZnOadded LMS ceramics are shown in figure 3. Sharp and intense peaks are formed at $521 \mathrm{~cm}^{-1}$ for LMS and LMS$\mathrm{ZnO}$ ceramic systems. These peaks imply the $\mathrm{Si}-\mathrm{O}-\mathrm{Si}$ bond thus confirming the formation of silicate. Considerable extra peaks are not present in the LMS-ZnO systems. Hence the FT-Raman spectra are in agreement with the corresponding XRD patterns.

Figure 4 shows the relative density and dielectric constant values obtained with the ceramic samples. From the figure, it is clear that density and dielectric constant show identical trends with respect to $\mathrm{ZnO}$ addition. Among all the samples pristine LMS achieved the highest relative density of $90.6 \%$ with a dielectric constant of 5.73. But, this ceramic needed more duration for sintering $\left(6 \mathrm{~h}\right.$ at $\left.1100^{\circ} \mathrm{C}\right)$. Reducing the duration of sintering to $4 \mathrm{~h}$ in LMS ceramics, 0.5 and $1 \mathrm{wt} \%$ $\mathrm{ZnO}$ addition resulted only in 85.8 and $88.2 \%$ of theoretical density. The highest relative density of pure LMS can be attributed to the way of mixing and sintering duration employed for LMS ceramics. Pure LMS was mixed through roll milling whereas $\mathrm{ZnO}$ was added to pure LMS through mortar grinding. But, addition of $\mathrm{ZnO}$ helped in the complete grain growth and the formation of uniform grain boundaries which is evident from the SEM results. Roll milling,

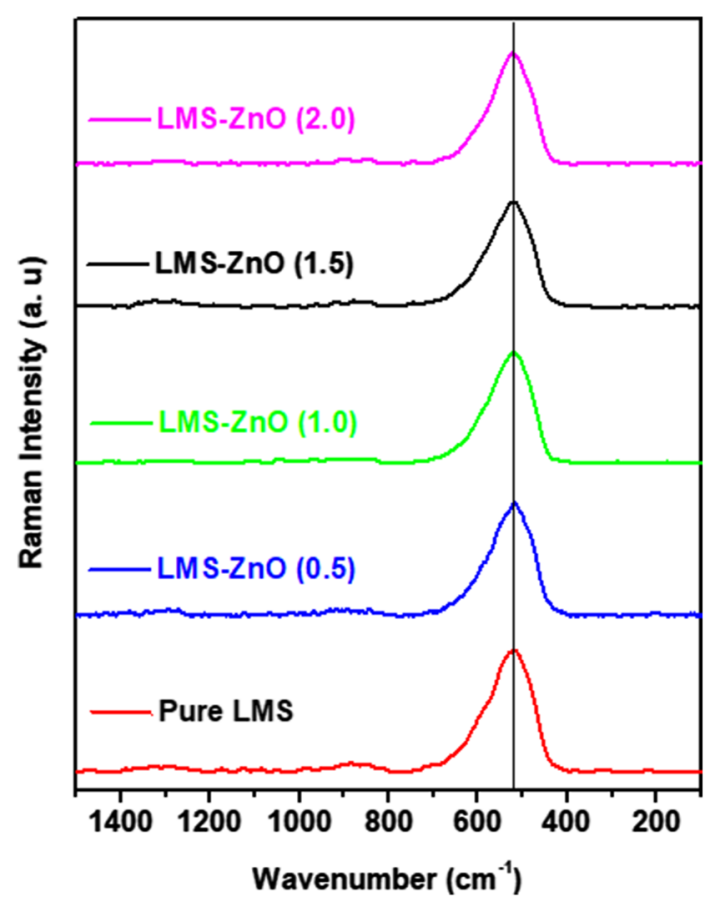

Figure 3. FT-Raman spectra of pure LMS and LMS-ZnO ceramics.

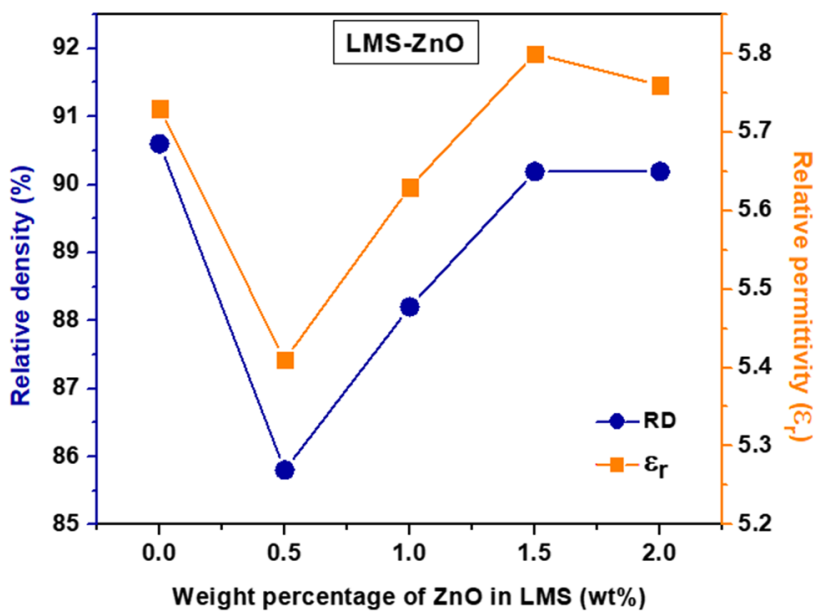

Figure 4. Relative density and dielectric constant values of pure LMS and LMS-ZnO ceramics.

which is an efficient way of mixing the starting materials in a stoichiometric ratio, may be applied to increase the density of LMS-ZnO ceramics. The microstructures corresponding to these ceramics in figure 2 showed porosity and incomplete densification. The neck-formation has been quite good but without sufficient grain coarsening. Because of this poor densification, relative permittivity of 0.5 and $1 \mathrm{wt} \% \mathrm{ZnO}$-added LMS ceramics exhibited relatively lower dielectric constants of 5.41 and 5.63, respectively compared to the pristine sample. But, addition of $\mathrm{ZnO} 1.5$ and $2 \mathrm{wt} \%$ in LMS ceramics achieved nearly the same density (90.2\% relative density) as 
that of pristine samples heated for $6 \mathrm{~h}$. It can be seen in figure 4 that the dielectric constant values of 1.5 and $2 \mathrm{wt} \%$-added LMS ceramics showed identical values with that of pristine ceramics.

Figure 5 displays the microwave dielectric Quxf of LMS and LMS-ZnO ceramics measured by a TE01 $\delta$ mode dielectric resonance technique at $12 \mathrm{GHz}$. The density and porosity of ceramics are really important in the quality factor of ceramics. Since the quality factor is the inverse of dielectric loss, the loss contributed by porosity, secondary phases or segregation and other microstructural defects decrease this parameter. Quxf initially decreases with $\mathrm{ZnO}$ addition like bulk density and then increases. Pristine LMS and LMS with $\mathrm{ZnO}$ (1 wt\%) show almost similar Quxf values. With further $\mathrm{ZnO}$ addition, the Quxf increases reaching the highest $(24,000 \mathrm{GHz})$ for $2 \%$ $\mathrm{ZnO}$ addition to LMS ceramic. From the HRSEM images (figure $2 \mathrm{a}-\mathrm{d}$ ) it is obvious that the LMS-ZnO (2 wt\%) sample contained homogenous grain growth and cohesive neck formations with less porosity. This fine microstructural feature has led to the highest quality factor. While comparing figures 2, 4 and 5, it is comprehensible that the density and microwave dielectric properties of LMS-ZnO (0.5 wt \%) were negatively affected by the presence of irregular grains, pores and micro-cracks leading to the lowest Quxf value. Thus, addition of 1.5 and $2 \mathrm{wt} \%$ of $\mathrm{ZnO}$ enhanced the microwave dielectric properties of LMS. The appearance of pores and cracks in the 0.5 and $1 \mathrm{wt} \%$ LMS-ZnO samples deteriorated microstructural density thereby reducing the quality factor and dielectric constant values. Since the dielectric properties of LMS are improved for the higher concentrations of $\mathrm{ZnO}$, this additive can be used in various concentrations with LMS for achieving a high quality factor. The presence of impurities in raw materials and contagion in the course of milling can cause less quality factor. Density of the final product can be enhanced by increasing the mixing time. An optimized milling duration can achieve uniform grain distribution thereby providing a dense microstructure. The right balance of these factors should be maintained to obtain a better quality ceramics. Variation in the dielectric constant is caused by the strength of bonding where increased bond strength lessens the relative permittivity value. Covalently bonded elements inside the LMS ceramic are the reason for decreased dielectric constants. The microwave dielectric properties of the LMS and LMS-ZnO ceramic systems prove that these are suitable candidates for substrate applications. Table 1 shows the density, relative density, dielectric constant, tangent loss and quality factor of the LMS and LMS-ZnO ceramic systems. Variation in the microwave dielectric properties of LMS upon the addition of different compounds is shown in figure 6 [35]. LMS with $\mathrm{ZnO}$ exhibits the lowest dielectric loss value and a below 10 dielectric constant value which indicates its capability to be used as an additive.

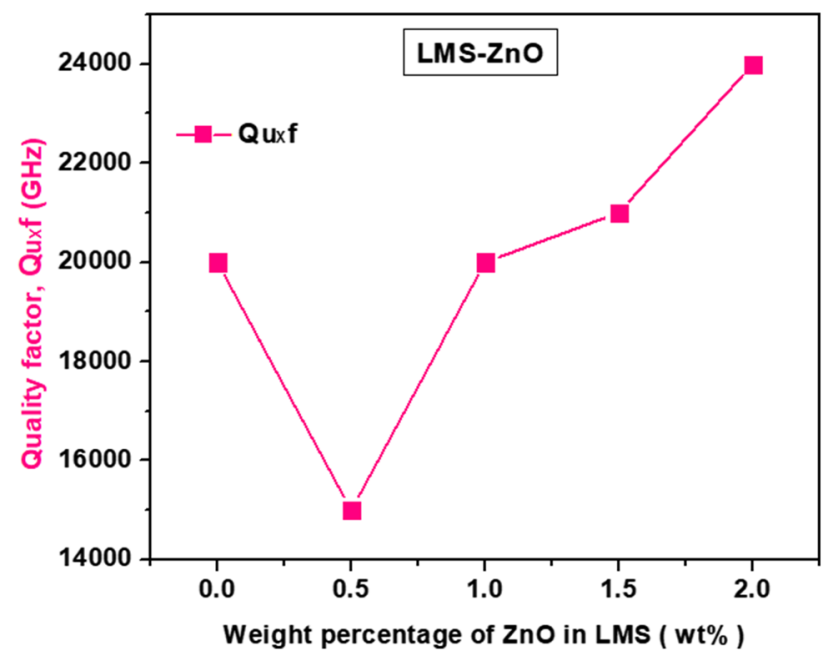

Figure 5. Quality factor of pure LMS and LMS-ZnO ceramics.

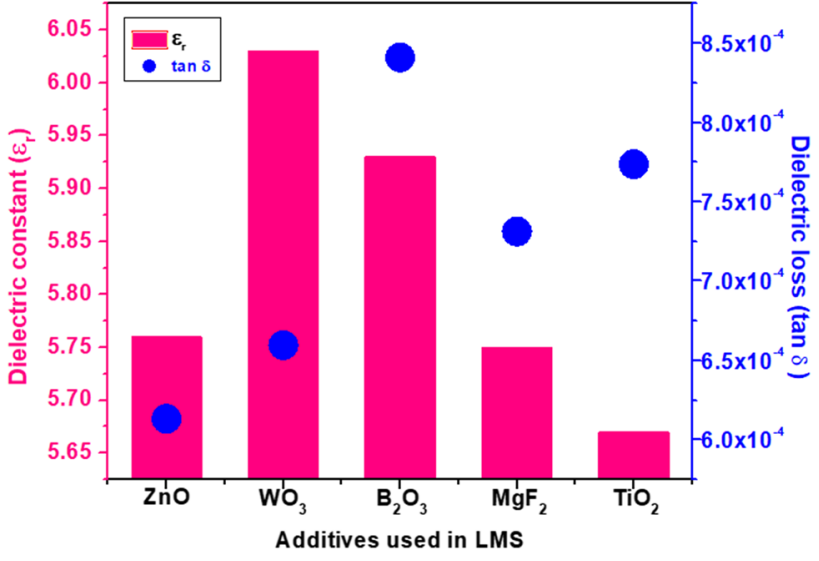

Figure 6. Comparison chart on the dielectric properties of LMS ceramics with different additives.

Table 1. Relative density and microwave dielectric properties of LMS and LMS-ZnO ceramics.

\begin{tabular}{lcccc}
\hline Sl. No. & ZnO in LMS (wt $\%)$ & Relative density $(\%)$ & Dielectric constant $\left(\varepsilon_{\mathrm{r}}\right)$ & Quality factor $(\mathrm{Quxf}) \mathrm{GHz}$ \\
\hline 1 & 0.0 & 90.6 & 5.73 & 20,000 \\
2 & 0.5 & 85.8 & 5.41 & 15,000 \\
3 & 1.0 & 88.2 & 5.63 & 20,000 \\
4 & 1.5 & 90.2 & 5.8 & 21,000 \\
5 & 2.0 & 90.2 & 5.76 & 24,000 \\
\hline
\end{tabular}




\section{Conclusion}

An effect of $\mathrm{ZnO}$ addition on the microwave dielectric properties of LMS was examined. LMS-ZnO with 1.5 and $2 \mathrm{wt} \% \mathrm{ZnO}$ showed a relatively higher quality factor among all ceramic systems. Pure LMS ceramic achieved the highest relative density of $90.6 \%$ and a Quxf value higher than that of the $0.5 \mathrm{wt} \% \mathrm{LMS}-\mathrm{ZnO}$ system due to the long duration of sintering temperature. The quality factor improved with the increasing weight percentage of $\mathrm{ZnO}$ in LMS. Also, relative permittivity exhibited by all the ceramic systems was $<10$. $\mathrm{ZnO}$ as an additive, thus, enhances the quality factor of LMS. Hence, LMS-ZnO ceramics can be investigated for higher concentrations of $\mathrm{ZnO}$ with the aim of obtaining the enhanced microwave dielectric properties apposite for substrate applications. LMS-ZnO $(2 \mathrm{wt} \%)$ ceramic showed the good dielectric properties of Quxf $=24,000 \mathrm{GHz}$ and $\varepsilon_{\mathrm{r}}=5.76$ at $12 \mathrm{GHz}$ while it acquired $90.2 \%$ of theoretical density. ZnO-addition is beneficial in LMS ceramics in reducing the sintering duration and achieving $20 \%$ or more quality factor.

\section{Acknowledgements}

The authors express their thanks to SRM University, Chennai, India, Advanced Materials and Ceramic Division, Vikram Sarabhai Space Centre, Trivandrum, India, and Department of Science and Technology (DST), SAIF-IIT Madras, India, for providing assistance to complete this research work.

\section{References}

[1] Pozar D M 2009 Microwave engineering (Hoboken, New Jersey, United States: Wiley)

[2] Sorrentino R and Bianchi G 2010 Microwave and RF engineering (Hoboken, New Jersey, United States: Wiley)

[3] Jones G A, Layer D H and Osenkowsky T G 2013 National association of broadcasters engineering handbook: NAB engineering handbook (Abingdon, United Kingdom: Taylor \& Francis)

[4] George S, Sajith V, Sebastian M T, Raman S and Mohanan P 2011 J. Adv. Dielectr. 1209

[5] George S, Anjana P S, Deepu V N, Mohanan P and Sebastian M T 2009 J. Am. Ceram. Soc. 921244
[6] Reaney I M and Iddles D 2006 J. Am. Ceram. Soc. 892063

[7] Sebastian M T 2010 Dielectric materials for wireless communication (Amsterdam, The Netherlands: Elsevier)

[8] Singh N, Kumar P and Rai R 2011 Adv. Mater. Lett. 2200

[9] Sunny A, Lazer K A, Manu K M, Surendran K P and Sebastian M T 2013 J. Alloys Compd. $\mathbf{5 5 2} 83$

[10] George S and Sebastian M T 2010 J. Eur. Ceram. Soc. 302585

[11] George S and Sebastian M T 2010 J. Am. Ceram. Soc. 932164

[12] Kolodiazhnyi T 2012 J. Eur. Ceram. Soc. 324305

[13] Chen X, Zhang W, Bai S and Du Y 2013 Ceram. Int. 396355

[14] Muhammad R, Iqbal Y, Rambo C R and Khan H 2014 Int. J. Mater. Res. 105431

[15] Thomas D, Rethika K and Sebastian M T 2012 J. Mater. Sci.: Mater. Electron. 231268

[16] Hu Y, Wei D, Fu Q, Zhao J and Zhou D 2012 J. Eur. Ceram. Soc. 32521

[17] Sasikala T, Suma M, Mohanan P, Pavithran C and Sebastian M T 2008 J. Alloys Compd. 461555

[18] Nguyen N H, Lim J B, Nahm S, Paik J H and Kim J H 2007 J. Am. Ceram. Soc. 903127

[19] Wang H, Zhang Q, Yang H and Sun H 2008 Ceram. Int. 34 1405

[20] Song K and Chen X 2008 Mater. Lett. 62520

[21] Sebastian M T and Jantunen H 2008 Int. Mater. Rev. 5357

[22] Li J, Tian Z, Yao L, Ran S and Fan C 2017 Ceram. Int. 43 15793

[23] Liu C, Zhang H, Wang G, Zhou T, Su H, Jia L et al 2017 Mater. Res. Bull. 9316

[24] Redozubov S, Kartenko N, Gaidamaka I and Nenasheva E 2016 Inorg. Mater. 521166

[25] Zhang P, Wang T, Xia W and Li L 2012 J. Alloys Compd. 5351

[26] Agathopoulos S 2012 J. Ceram. Soc. Jpn. 120233

[27] Tan C, Tan Y, Ramesh S and Yap B 2017 IOP Conf. Series. Mater. Sci. Eng. 206012051

[28] Nikzad L, Majidian H, Ghofrani S and Ebadzadeh T 2018 Int. J. Appl. Ceram. Technol. 15569

[29] Lu X, Huang B, Chen T, Fu Z, Wang Z, Wang L et al 2016 J. Mater. Sci.: Mater. Electron. 272687

[30] Muccillo R, Muccillo E, Andrade T and Oliveira O 2017 J. Therm. Anal. Calorimetry 1301791

[31] Guo Y and Zhang Y 2017 J. Mater. Sci.: Mater. Electron. 28 7248

[32] Xie F, Jia L, Xu F, Li J, Liao Y, Li D et al 2018 J. Mater. Sci.: Mater. Electron. 295885

[33] Weng Z, Guan R and Xiong Z 2017 J. Alloys Compd. 6953517

[34] Li L, Li J, Zhang N, Cai Z, Zhang B and Luo W 2017 J. Mater. Sci.: Mater. Electron. 289763

[35] Rose A, Masin B, Sreemoolanadhan H, Ashok K and Vijayakumar T 2018 Appl. Surf. Sci. 44996 